\title{
Bcl-2 expression in sputum eosinophils in patients with acute asthma
}

An-Soo Jang, Inseon-S Choi, Soong Lee, Jeong-Pyeong Seo, Seung-Won Yang, Chang-Soo Park

\begin{abstract}
Background-Eosinophils play a pivotal role in asthmatic airway inflammation. Apoptosis is thought to be critically relevant in promoting the clearance of inflammatory cells and the resolution of inflammation. Bcl-2 inhibits apoptosis in cellular systems. A study was undertaken to determine whether bcl-2 expression in sputum reflects the clinical severity of patients with asthma. The relationship between bcl-2 expression in sputum eosinophils and eosinophil activation markers such as interleukin (IL) -5 and eosinophil cationic protein (ECP) levels in sputum supernatant was evaluated.
\end{abstract}

Methods-Sputum was obtained from 18 patients with asthma. Fresh expectorated sputum separated from saliva was treated with an equal volume of dithiothreitol $0.1 \%$, cytospun for cell differentials and bcl-2 stain, and the supernatant was collected for biochemical assay. Bcl-2+ eosinophils were stained using immunocytochemistry, ECP was measured by fluoroimmunoassay, and IL-5 was detected by sandwich enzyme linked immunosorbant assay.

Results-Twelve patients with severe or life threatening asthma had more bcl-2+ eosinophils (mean difference $46.8 \%$ (95\% CI 27.0 to 66.6), p<0.01) and a higher ECP level $(p<0.01)$ in the sputum than those with mild to moderate asthma $(n=6)$. IL-5 was frequently detected in patients with severe or life threatening asthma $(11 / 12$ versus $1 / 6, p<0.01)$. There was a significant positive correlation between bcl-2+ eosinophils and ECP levels $(r=$ $0.61, \mathrm{p}<0.01)$ and between bcl-2+ eosinophils and IL-5 levels $(r=0.83, \mathrm{p}<0.01)$. There was a significant negative correlation between bcl-2+ eosinophils and $\mathrm{FEV}_{1} I$ FVC $(r=-0.54, \mathrm{p}<0.05)$.

Conclusion-The increased expression of bcl-2 in eosinophils from sputum of subjects with severe asthma suggests that bcl-2 may prolong survival and decrease apoptosis of airway eosinophils in asthma. (Thorax 2000;55:370-374)

Keywords: eosinophils; apoptosis; asthma; sputum; bcl-2

Eosinophils are known to play a pivotal role in asthmatic airway inflammation. Infiltration of eosinophils into the bronchial wall and respiratory epithelial damage are two distinctive features of asthma. ${ }^{1}$ These bronchial changes involve four steps-namely, enhanced eosinophil production, recruitment of lung tissue, activation of eosinophils, and release of mediators.

The prolongation of eosinophil survival is important in the pathogenesis of asthmatic airway inflammation. Apoptosis plays a central part in normal tissue homeostasis as well as having a role in various clinical diseases characterised by either increased or decreased cell survival. ${ }^{2}$ It is thought to be critically important in promoting the clearance of inflammatory cells and the resolution of inflammation. Apoptosis of eosinophils may be clinically relevant in asthma, promoting the removal of airway eosinophils and contributing to clinical improvement. Among apoptosis suppressing genes, bcl-2 prevents apoptosis either through altering cell cycle rates or by activating antioxidant associated mechanisms. ${ }^{2}{ }^{3}$

Interleukin (IL)-5, a cytokine that attracts, activates, and prolongs the survival of eosinophils, is important in causing eosinophilic inflammation in the asthmatic airway and contributing to eosinophil viability in the sputum of asthmatic patients during attacks. ${ }^{4}$ It inhibits eosinophil apoptosis by upregulation of bcl- $2 .^{5}$

Vignola et $a l^{6}$ reported that bcl-2+ cells in bronchial biopsy specimens from patients with asthma were higher than those from control subjects and from subjects with chronic bronchitis; the number of bcl-2+ cells correlated with the severity of the disease. Skin biopsy specimens from patients with hypereosinophilic syndrome have also been reported to express bcl-2 protein. ${ }^{7}$

This study was undertaken to determine whether bcl-2 expression in sputum reflects the clinical severity of patients with asthma. The relationship between bcl-2 expression in sputum eosinophils and eosinophil activation markers such as IL-5 and eosinophil cationic protein (ECP) in sputum supernatant was evaluated.

\section{Methods}

SUBJECTS

Eighteen patients with asthma were recruited from the Division of Allergy, Department of Internal Medicine, Seonam University and Chonnam University Hospital between February and August 1999. The diagnosis of asthma had been previously established in 13 patients by their symptoms of recurrent episodic wheezing, cough, and/or dyspnoea, and either by methacholine airway hyperresponsiveness in 
10 patients or by an improvement of $15 \%$ after salbutamol $200 \mu \mathrm{g}$ (when the forced expiratory volume in one second $\left(\mathrm{FEV}_{1}\right)$ was $<75 \%$ predicted) in three patients. In the five remaining patients the diagnosis was confirmed by an improvement in the $\mathrm{FEV}_{1}(15 \%)$ following anti-asthma therapy. No subject had respiratory infection for four weeks before the study. Seonam University Hospital Research Committee approved the study and all subjects signed the informed consent forms.

\section{STUDY DESIGN}

Eosinophils and eosinophil activation markers in sputum were measured in asthmatic patients. A questionnaire on symptoms and medications was given to all patients and a spirometric test was performed. Sputum was then collected and skin prick tests and a methacholine provocation test were performed. The clinical severity of asthma was classified according to the International Consensus Report. ${ }^{8}$ Asthma exacerbations were graded by symptoms (breathlessness, talking, and alertness), signs (respiratory rate, use of accessory muscles, wheeze, pulse/min, and pulsus paradoxus), and functional assessment (peak expiratory flow rate, arterial blood gas analysis). A person blinded to the clinical characteristics of the subjects performed the sputum measurements.

Spirometric tests were performed according to the American Thoracic Society standards ${ }^{9}$ using a SensorMedics 2200 spirometer (Cardiopulmonary Care Company, California, USA). The representative values for forced vital capacity (FVC) and forced expiratory volume in one second $\left(\mathrm{FEV}_{1}\right)$ were selected according to the Intermountain Thoracic Society criteria ${ }^{10}$ and the reference values were taken from the reports by Choi et a ${ }^{11}$ with some modifications. Methacholine challenge tests were carried out using a modification of the method described by Chai et $a l^{12}$ and the results were expressed as $\mathrm{PC}_{20}$ in non-cumulative units. Allergy skin prick tests were performed using 55 common allergen extracts. The wheal size was recorded at 15 minutes and scored as follows: $0=$ no different from control, $+=$ 1-2 $\mathrm{mm}$ larger than control, $++=3-5 \mathrm{~mm}$ larger than control, $+++=6-8 \mathrm{~mm}$ larger than control, $++++=>8 \mathrm{~mm}$ larger than control. The total number of pluses was added. Atopy was defined as an atopy score of $>1+$.

SPUTUM INDUCTION AND PROCESSING

Sputum was induced only when it could not be produced spontaneously. The sputum induction was performed as a modification of the method described by Fahy et al. ${ }^{13}$ All subjects were premedicated with two puffs of inhaled salbutamol $(200 \mu \mathrm{g})$. Subjects inhaled 3\% hypertonic saline solution aerosols generated by an ultrasonic nebuliser (NE-U03, OMRON Co, Tokyo, Japan) with a maximum output of $0.15-0.3 \mathrm{ml} / \mathrm{min}$ and a mass median aerodynamic diameter of $4.5 \mu \mathrm{m}$. Hypertonic saline was inhaled for 15-20 minutes according to the severity of the asthma until an adequate volume of sputum was expectorated. Subjects were asked to rinse their mouth and blow their nose to minimise contamination with saliva and postnasal drip. They were encouraged to cough deeply and frequently during hypertonic saline inhalation. They were instructed to cough the sputum into a sterile plastic container. The sample volumes and duration of sputum induction were recorded. $\mathrm{FEV}_{1}$ was measured before, during, and after induction of sputum. Sputum induction was stopped if the $\mathrm{FEV}_{1}$ fell by $\geqslant 15 \%$.

The appearance of the sputum was recorded as mucoid, mucopurulent, or purulent. Sputum was selected from saliva and processed within two hours. The method of sputum examination described by Popov et $a l^{14}$ was modified. ${ }^{12}$ Sputum was treated by adding equal volumes of $0.1 \%$ dithiothreitol (Sputalysin 10\%; Gibco BRL, Grandisland, New York, USA) followed by equal volumes of Dulbecco's phosphate buffered saline (D-PBS). The sample was then mixed gently and placed in a shaking water bath at $37^{\circ} \mathrm{C}$ for 15 minutes to ensure complete homogenisation. The sample was removed from the water bath periodically for further brief gentle mixing. The suspension was filtered through gauze $(1 \mathrm{~mm}$ pore size), the filtrate was centrifuged at 1500 rpm for 10 minutes, and the supernatant was aspirated and stored in Eppendorf tubes at $-70^{\circ} \mathrm{C}$ for later assay. The cell pellet was resuspended in $1000 \mu \mathrm{l} \mathrm{D}-\mathrm{PBS}$ and the total number of non-squamous cells was counted in a modified Neubauer haemocytometer. The cell suspension was adjusted to $0.5 \times 10^{5} / \mathrm{ml}$ and then $50 \mu \mathrm{l}$ of cell suspension was placed in a Sakura cytocentrifuge (Model CF-127, Tokyo, Japan). Two coded cytospins were prepared at $600 \mathrm{rpm}$ for five minutes. One slide was air dried and stained with Trypan blue stain (GibcoBRL, Grand Island, New York, USA) and two others were stained with Diff-Quick (Kookje Scientific Products, Tokyo, Japan). Another two slides prepared for immunocytochemical analysis were air dried for 10 minutes, fixed in $0.25 \%$ formalin for five minutes at $4^{\circ} \mathrm{C}$, wrapped in pairs in foil and stored at $-70^{\circ} \mathrm{C}$. Cell differential counts of 400 non-squamous cells were performed on DiffQuick stained slides by two investigators who did not know the subject's clinical history, and the results were expressed as a percentage of the total non-squamous cell count.

IMMUNOCYTOCHEMICAL ANALYSIS OF BCL-2 Immunocytochemical analysis was performed on sputum cytospin slides with Probe On Plus slide (Fisher Scientific, Pittsburgh, Pennsylvania, USA). All steps in the staining procedure were done at $50^{\circ} \mathrm{C}$ in a Microprobe system taking advantage of capillary gap action ${ }^{15}$ produced by two approximated Probe On Plus slides. The primary antibody was applied for 15 minutes with anti bcl-2 antibody (Dako, Copenhagen, Denmark). Antigen/antibody complexes were detected with a goat antimouse IgG (Sigma, St Louis, Missouri, USA) for 10 minutes followed by avidine alkaline phosphatase (Dako) ${ }^{16}$ for seven minutes after blocking endogenous alkaline phosphatase 
Table 1 Characteristics of patients with asthma

\begin{tabular}{lll}
\hline & $\begin{array}{l}\text { Mild to moderate } \\
\text { asthma }(n=6)\end{array}$ & $\begin{array}{l}\text { Severe to life } \\
\text { threatening asthma } \\
(n=12)\end{array}$ \\
\hline Age (years) & $41.3(3.2)$ & $48.8(5.5)$ \\
Sex (M/F) & $3 / 3$ & $8 / 4$ \\
Smoker (ex) & $1(1)$ & $3(5)$ \\
IgE (IU/ml) & $252.2(91.6)$ & $777.3(425.4)$ \\
PC $_{20}(\mathrm{mg} / \mathrm{ml})$ & $2.03(1.81)$ & $1.11(0.53)$ \\
$\mathrm{FEV}_{1}(\% \mathrm{pred})$ & $79.2(4.5)$ & $63.4(4.1)^{\star}$ \\
FVC $(\% \mathrm{pred})$ & $75.1(5.0)$ & $68.1(8.5)$ \\
FEV $_{1} / \mathrm{FVC}(\%)$ & $80.0(9.6)$ & $57.9(3.8)^{\star}$ \\
\hline
\end{tabular}

Data are expressed as mean (SE).

$\mathrm{PC}_{20}=$ concentration of methacholine provoking a fall of $20 \%$ in $\mathrm{FEV}_{1}$ if the $\mathrm{FEV}_{1} \geqslant 75 \% ; \mathrm{FEV}_{1}=$ forced expiratory volume in one second; FVC = forced vital capacity. ${ }^{\star} \mathrm{p}<0.01$.

activity in Redusol (Biomeda, Foster City, California, USA) for two minutes. The chromogen reaction consisted of Fast Red TR Salt (Research Genetics, Huntsville, Alabama, USA) for 10 minutes followed by 30 seconds of haematoxylin. Bcl-2 antibodies were diluted in primary antibody diluent (Research Genetics) at the final concentration of $5 \mu \mathrm{g} / \mathrm{ml}$. Positive controls for bcl-2 consisted of known positive samples of human spleen included with each staining reaction. Negative controls consisted of staining without the primary antibody. All slides were examined using standard light microscopy by two observers and scored as percentage of positive eosinophils over total eosinophils. The intensity of the staining reaction was not quantified. The results were expressed as percentage of positively stained cells over total eosinophils.

ECP AND IL-5 MEASUREMENT

The concentration of ECP in $400 \mu$ thawed supernatant was determined by fluoroimmunoassay (UniCAP system, Pharmacia AB, Uppsala, Sweden). IL-5 was measured by quantitative sandwich enzyme immunoassay (Quantikine; R\&D System Inc). Samples were analysed in duplicates. The limits of detection for the ECP and IL-5 assays were $2.0 \mu \mathrm{g} / 1$ and $3 \mathrm{pg} / \mathrm{ml}$, respectively.

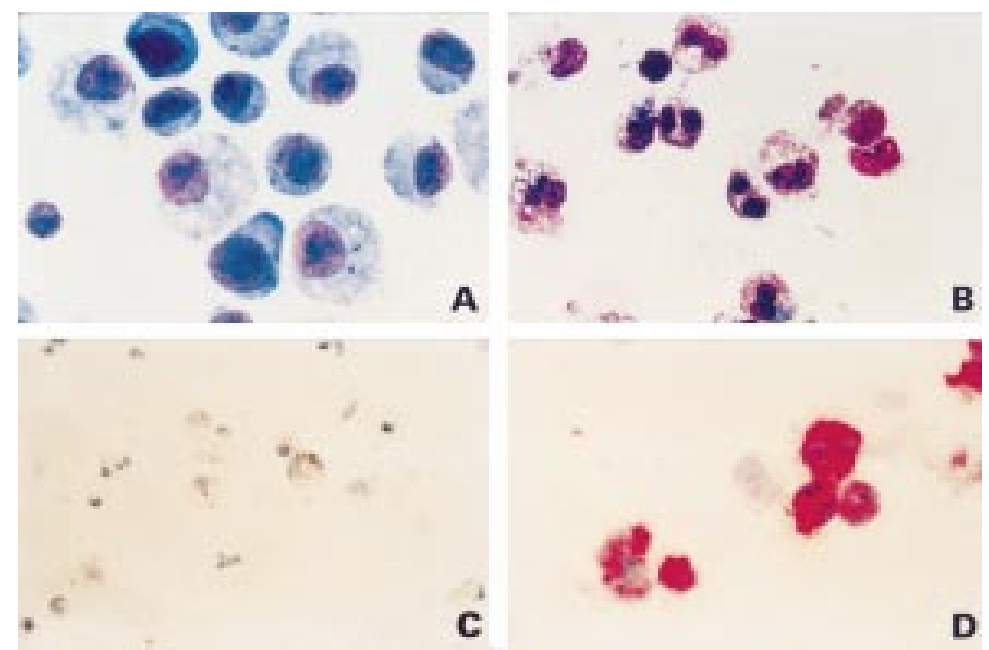

Figure 1 Sputum cells stained with Diff-Quick in (A) control and (B) asthmatic subjects and eosinophils stained with anti-bcl-2 antibody by immunocytochemistry in negative control from (C) asthmatic subjects without the primary antibody and (D) asthmatic subjects. Eosinophil staining with bcl-2 antibody in asthmatic patients shows cytoplasmic red staining.
STATISTICAL ANALYSIS

All data were analysed using the SPSS version 7.5 for Windows. Data are expressed as mean (SE). Inter-group comparisons were assessed by a non-parametric method using the MannWhitney $U$ test. The correlation between variables was examined by Spearman rank correlation coefficient. A p value of less than $5 \%$ was regarded as statistically significant.

\section{Results}

The characteristics of the study subjects are shown in table 1. Patients with severe to life threatening asthma had significantly lower $\mathrm{FEV}_{1}$ and $\mathrm{FEV}_{1} / \mathrm{FVC}$ than those with mild to moderate persistent asthma.

\section{DIFFERENTIAL SPUTUM COUNTS}

Eighteen sputum samples from asthmatic patients were analysed. The sputum had less than $30 \%$ squamous epithelial cells and more than $85 \%$ cell viability. The proportion of eosinophils was significantly higher in patients with severe or life threatening asthma than in those with mild to moderate asthma (49.8 (6.5)\% versus $10.3(4.1) \%, p<0.01)$, whereas the proportion of macrophages was lower (26.4 $(5.5) \%$ versus $59.5(11.8) \%, \mathrm{p}<0.01)$. No significant differences were noted between the two groups of patients in either total cell count $\left(8.1(3.4) \times 10^{5} / \mathrm{ml}\right.$ versus $\left.5.0(0.9) \times 10^{5} / \mathrm{ml}\right)$ or the absolute or relative numbers of lymphocytes and epithelial cells.

BCL-2+ EOSINOPHILS, ECP, AND IL-5

Positive staining eosinophils with bcl-2 antibody (fig 1) were significantly increased in the cytoplasm of eosinophils from patients with severe or life threatening asthma compared with those with mild to moderate asthma (49.3 (6.5)\% versus $2.5 \quad(0.5) \%$, $\mathrm{p}<0.01$, fig 2). The mean difference was $46.8 \%$ (95\% CI 27.0 to 66.6$)$. The sputum level of ECP was significantly increased in patients with severe to life threatening asthma

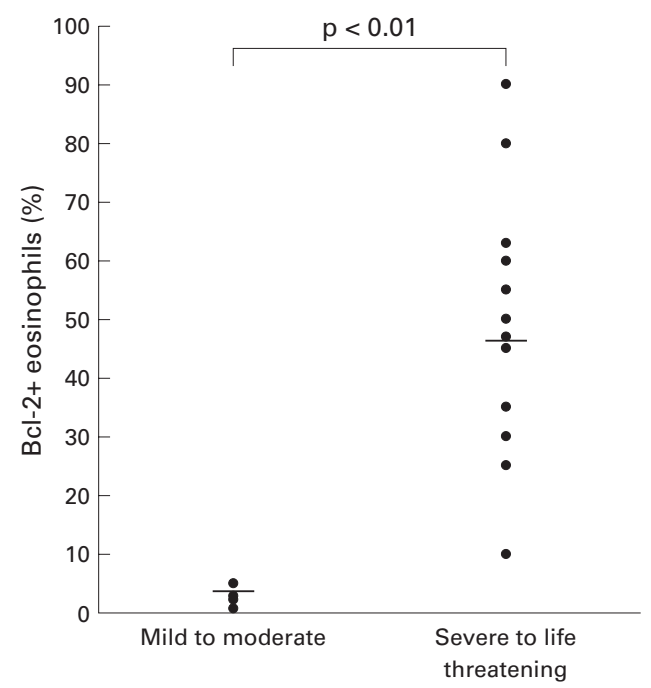

Figure 2 Percentage of bcl-2+ eosinophils in the sputum of patients with mild to moderate asthma and with severe to life threatening asthma. The results are expressed as the percentage of bcl-2 expressing eosinophils in the sputum. Horizontal bars indicate mean values. 


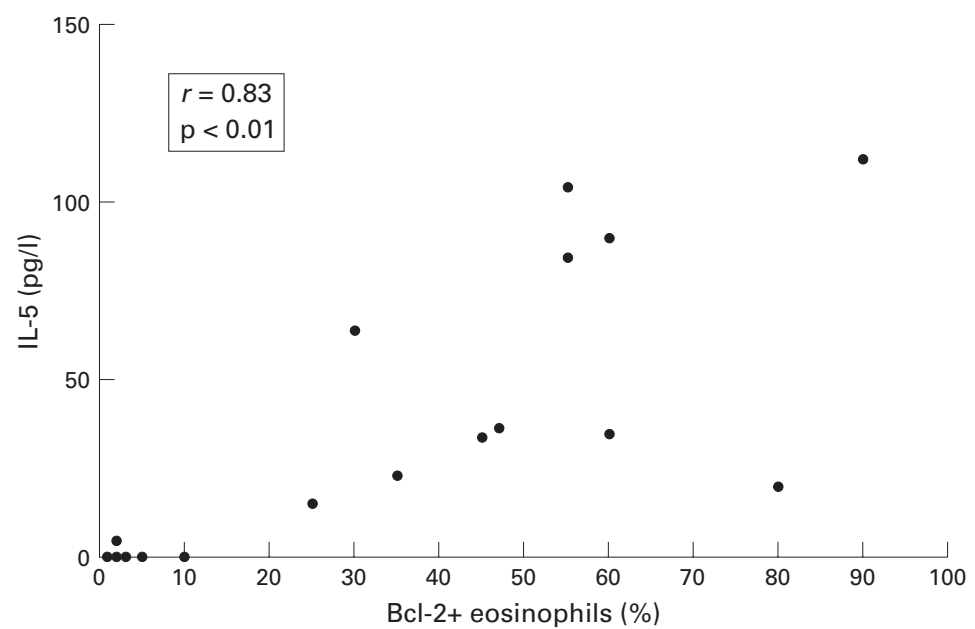

Figure 3 Correlation between bcl-2+eosinophils in the sputum and IL-5 levels in the sputum supernatant.

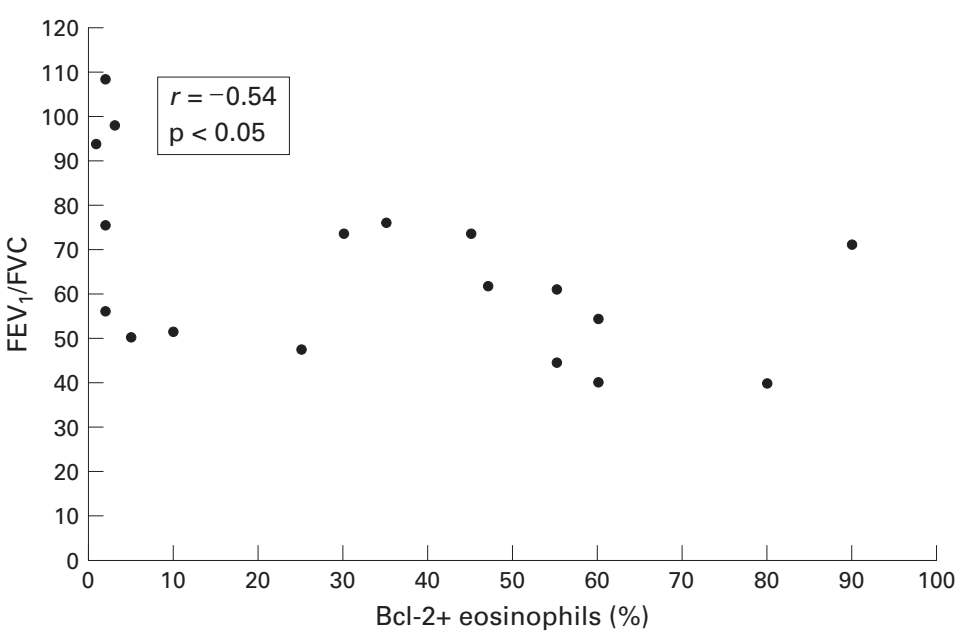

Figure 4 Correlation between bcl-2+eosinophils in the sputum and FEV /FVC.

compared with that in patients with mild to moderate asthma (1746.1 (293.0) $\mu \mathrm{g} / \mathrm{l}$ versus $618.4(465.7) \mu \mathrm{g} / \mathrm{l}, \mathrm{p}<0.05)$. IL-5 was more frequently detected in patients with severe to life threatening asthma (11/12 versus $1 / 6$, $\mathrm{p}<0.01)$.

CORRELATIONS BETWEEN BCL-2+ EOSINOPHILS AND CLINICAL FINDINGS

There was a significant positive correlation between bcl-2+ eosinophils and ECP levels $(r$ $=0.61, \mathrm{p}<0.01)$. The proportions of sputum eosinophils were positively correlated with bcl-2+ eosinophils $(r=0.58, \mathrm{p}<0.01)$. There was also a significant positive correlation between bcl-2+ eosinophils and IL-5 levels $(r=$ 0.83 , p $<0.01$, fig 3 ) and a significant negative correlation between bcl-2+ eosinophils and $\mathrm{FEV}_{1} / \mathrm{FVC}(r=-0.54, \mathrm{p}<0.05$, fig 4$)$. No correlation was noted between bcl $2+$ eosinophils in the sputum and atopy score or non-specific airway hyperresponsiveness.

\section{Discussion}

Inhibition of eosinophil apoptosis may play an important role in chronic airway inflammation. In this study we found that anti-apoptotic bcl-2 protein was increased in patients with severe asthma compared with those with mild asthma. The evaluation of bcl-2 expression in sputum eosinophils may be a useful marker for monitoring airway inflammation in asthma.

Asthma results from complex interactions between inflammatory cells, mediators, and the cells and tissues of the airways. ${ }^{17}$ Eosinophils are known to play a major part in asthmatic airway inflammation. They are cells of granulocyte lineage formed in the bone marrow which move through the circulation and migrate into the airways. The bone marrow transit time in humans is about 4.3 days. In peripheral blood the eosinophil has a half life of about 18 hours. Recruitment from the blood to the airway initially involves adherence of eosinophils to endothelial cells and subsequent migration into an inflammatory site. Inflammatory cytokines such as IL-1, IL-4, IL-5, interferon (IFN)- $\gamma$, and tumour necrosis factor (TNF)- $\alpha$ enhance the interaction between inflammatory cells and endothelial cells, and endothelial cells induce increased expression of adhesion molecules such as intercellular adhesion molecule 1 and vascular cell adhesion molecule, and transendothelial migration of eosinophils to inflamed tissues. ${ }^{18} 19$ In target lung tissue degranulation of eosinophilic granules such as ECP, major basic protein, eosinophil derived neurotoxin, and eosinophil peroxidase is fundamental to the proinflammatory role of eosinophils. These eosinophilic granular contents damage the airway epithelium, stimulate the release of histamine from mast cells, directly contract airway smooth muscle, and promote airway hyperresponsiveness. ${ }^{2021}$

As previously suggested, in this study the eosinophil activation markers IL-5 and ECP significantly increased in patients with severe asthma. This suggests that the higher percentage of eosinophils and increased levels of eosinophil activation markers measured in asthmatic sputum are due to recruitment of eosinophils and degranulation of eosinophils in target tissues.

Apoptosis or programmed cell death is an ordered process of fundamental biological importance which is as vital to homeostasis as are cellular proliferation, differentiation, and migration. $^{22} 23$ It is thought to be critically important in promoting the clearance of inflammatory cells and the resolution of inflammation. Apoptosis of eosinophils may be clinically relevant in asthma, promoting the removal of airway eosinophils and contributing to clinical improvement. IL-5, a cytokine that attracts, activates, and prolongs the survival of eosinophils, is very important in the pathogenesis of allergic inflammation and asthma. ${ }^{4}$ It has an inhibitory effect on eosinophil apoptosis by regulating bcl-2 expression. ${ }^{3}$ Dewson et a $2^{3}$ reported that cultivation of eosinophils in the presence of $100 \mathrm{pg} / \mathrm{ml}$ IL-5 for 24 hours significantly reduced apoptosis $(\mathrm{p}<0.01)$ to $10.7(2.5) \%$ compared with $46.8(7.4) \%$ in the absence of IL-5, suggesting that survival enhancing IL-5 modulates the expression of bcl-2 in vitro. We found that IL-5 was frequently detected in patients with severe to life threatening asthma and expression of bcl-2 
protein in sputum eosinophils was related to the severity of asthma, suggesting that IL-5 prolongs the survival of eosinophils. Overexpression of bcl-2 has been shown to prolong cellular survival in myeloid leukemia cell lines $^{2425}$ and neutrophils, ${ }^{26}$ as well as lymphoid cell lines, by blocking apoptosis.

Assessment of apoptosis ${ }^{2}$ is performed using methods that evaluate the physical changes of the cell by light and electron microscopy, as well as detection of the classical fragmentation of intracellular DNA. Alternative approaches include detection of DNA degradation using flow cytometric methods and evaluation of the expression of apoptosis genes with techniques such as in situ hybridisation or Northern blotting. In this study we used immunocytochemical staining to detect apoptosis protein. Bcl-2+ eosinophils were significantly more numerous in the cytoplasm of sputum eosinophils from patients with severe to life threatening asthma than in those with mild to moderate asthma. Other studies such as in situ hybridisation, reverse transcriptase polymerase chain reaction (RT-PCR), and western blotting are needed to confirm bcl-2 expression in asthmatic sputum eosinophils.

There was a significantly positive correlation between bcl-2+ eosinophils and ECP levels and the proportions of sputum eosinophils were positively correlated with bcl-2+ eosinophils. There was a significant negative correlation between bcl-2+ eosinophils and $\mathrm{FEV}_{1}$ / FVC. Taken together, expression of bcl-2 in the sputum eosinophils is a marker of severity of airway inflammation and clinical severity. The range of the study could have been extended either by looking at expression of bcl-2 in more detail, by determining the relationship between bcl-2 expressing eosinophils and apoptosis, or by investigating other apoptosis related proteins. Further studies will be required to evaluate changes in eosinophil apoptosis in the sputum of patients with asthma after treatment with an anti-inflammatory agent.

In summary, we examined the expression of bcl-2 protein in the sputum of patients with asthma and found more bcl- 2 protein in the sputum from patients with severe asthma than that from patients with mild asthma. These results suggest a possible contribution of bcl-2 protein to asthmatic airway inflammation, including inhibition of eosinophil apoptosis.

The authors thank Seog Chae Park and En Mee Park for their excellent assistance.
1 Frigas E, Gleich GJ. The eosinophil and the pathophysiol-

ogy of asthma. I Allergy Clin Immunol 1986;77:527-37.
Fleisher TA. Apoptosis. Ann Allergy Asthma Immunol 1997; Fleisher TA.

3 Salmon M, Pilling D, Borthwick NJ, Akbar AN. Inhibition of $T$ cell apoptosis: a mechanism for persistence in chronic inflammation. The Immunologist 1997;5/3:87-92.

4 Adachi T, Motojima S, Hirata A, et al. Eosinophil viabilityenhancing activity in sputum from patients with bronchial asthma: contribution of interleukin-5 and granulocyte/ macrophage colony-stimulating factor. Am $\mathcal{f}$ Respir Crit Care Med 1995;151:618-23.

5 Ochiai K, Kagami M, Matsumura R, et al. IL-5 but not interferon-gamma inhibits eosinophil apoptosis by upinterferon-gamma inhibits eosinophil apoptosis by upregulation

6 Vignola AM, Chanez P, Chiappara G, et al. Evaluation of apoptosis of eosinophils, macrophages, and T lymphocytes in mucosal biopsy specimens of patients with asthma and chronic bronchitis. $\mathcal{F}$ Allergy Clin Immunol 1999;103:56373.

7 Plötz SG, Dibbert B, Abeck D, et al. Bcl-2 expression by eosinophils in a patient with hypereosinophilia. F Allergy Clin Immunol 1998;102:1037-40.

8 Global Initiative for Asthma. Global strategy for asthma management and prevention. NHLBI/NIH workshop report. agement and prevention. NHLBI/NIH works

9 American Thoracic Society. Lung function testing: selection of reference values and interpretative strategies. Am Rev Respir Dis 1991;144:1202-18.

10 Morris AH, Kanner RE, Crapo RO, et al. Clinical pulmonary function testing: a manual of uniform laboratory procedures. 2nd ed. Salt Lake City: Intermountain Thoracic Society, 1984

11 Choi IS, Greville HW, Park KO. Addition of peak expiratory flow rate to the selection criteria of the representative spirometric result. Chonnam f Med Sci 1990;3:23-8.

12 Chai H, Farr RS, Froehlich LA, et al. Standardization of bronchial inhalation challenge procedures. I Allergy Clin Immunol 1975;56:323-7.

13 Fahy JV, Liu J, Wong $\mathrm{H}$, et al. Cellular and biochemical analysis of induced sputum from asthmatic and from analysis of induced sputum from asthmatic and from

14 Popov T, Gottschalk R, Kilendowwicz R, et al. The evaluation of a cell dispersion method of sputum examination. Clin Exp Allergy 1984;24:778-83.

15 Reed JA, Manahan LJ, Park CS, et al. Complete one hour immunocytochemistry based on capillary action. Biotechniques 1992; 13:434-43.

16 Guesdon JL, Ternynck T, Avrameas S. The use of avidin-biotin interaction in immunoenzymatic techniques. f Histochem Cytochem 1979;27:1131-9.

17 Expert Panel Report 2, National Asthma Education and Prevention Program: Guidelines for the diagnosis and management of asthma. National Heart, Lung, and Blood Institute. NIH Publication No. 97-4051A. 1997: 1-34.

18 Weller PF, Rand TH, Goelz SE, et al. Human eosinophil adherence to vascular endothelium mediated by binding to vascular cell adhesion molecule 1 and endothelium leukovascular adhesion molecule 1. Proc Natl Acad Sci USA cyte adhesion mole $1991 ; \mathbf{8 8}: 7530-3$.

19 Hansel TT, Walker C. The migration of eosinophils into sputum of asthmatics: the role of adhesion molecules. Clin Exp Allergy 1992;22: 345-56.

20 Gleich GJ. The eosinophil and bronchial asthma: current understanding. $\mathcal{F}$ Allergy Clin Immunol 1990;85:422-36.

21 Frigas E, Gleich GJ. The eosinophil and the pathophysiology of asthma. F Allergy Clin Immunol 1986;77:527-37.

22 Wyllie AH, Kerr JFR, Currie AR. Cell death. The significance of apoptosis. Int Rev Cytol 1980;68:251-306.

23 Dewson G, Walsh GM, Wardlaw AJ. Expression of Bcl-2 and its homologues in human eosinophils. Modulation by and its homologues in human eosinophils. Modulation

24 Delia D, Aiello A, Soligo D, et al. Bcl-2 proto-oncogene expression in normal and neoplastic human myeloid cells. expression in normal and

25 Naumovski L, Cleary ML. Bcl-2 inhibits apoptosis associated with terminal differentiation of HL-60 myeloid leukemia cells. Blood 1994;83:2261-7.

26 Lagasse E, Weissman IL. Bcl-2 inhibits apoptosis of neutrophils but not their engulfment by macrophages. $f$ Exp Med 1994;179:1047-52. 